\title{
Schottky Junctions Based on the ALD-ZnO Thin Films for Electronic Applications
}

\author{
T.A. Krajewski ${ }^{a, *}$, G. Luka ${ }^{a}$, P.S. Smertenko ${ }^{b}$, A.J. Zakrzewski ${ }^{a, c}, \mathrm{~K}$ DybKo $^{a}$, \\ R. JAkiela ${ }^{a}$, L. WAChNicki ${ }^{a}$, S. Gieraltowska ${ }^{a}$, B.S. Witkowski ${ }^{a}$, M. Godlewski ${ }^{a, c}$ \\ AND E. GUZIEWICZ ${ }^{a}$ \\ ${ }^{a}$ Institute of Physics, Polish Academy of Sciences, al. Lotników 32/46, 02-668 Warsaw, Poland \\ ${ }^{b}$ Institute of Semiconductor Physics, National Academy of Sciences of Ukraine, Pr. Nauki 45, 03028 Kyiv, Ukraine \\ ${ }^{c}$ Department of Mathematics and Natural Sciences College of Science, Cardinal Stefan Wyszyński University \\ Dewajtis 5, 01-815 Warsaw, Poland
}

The ZnO-based Schottky diodes revealing a high rectification ratio may be used in many electronic devices. This paper demonstrates several approaches to obtain a $\mathrm{ZnO}$-based Schottky junction with a high rectification ratio. The authors tested several methods such as: post-growth annealing of the ZnO layer, acceptor (nitrogen) doping, as well as the $\mathrm{ZnO}$ surface coating with a properly chosen dielectric material. The influence of these approaches on the diode's rectification ratio together with modeling based on the differential approach and thermionic emission theory are presented.

PACS: 68.55.ag, 73.50.-h, 73.50.Bk, 73.61.Ga, 81.15.-z, 81.15.Gh

\section{Introduction}

Zinc oxide $(\mathrm{ZnO})$ is presently tested for advanced electronic applications. For example, a $\mathrm{ZnO}$-based junction is considered as a selector in the new generation of $3 \mathrm{D}$ memory cells built in the so-called cross-bar architecture $[1,2]$. For this application a ZnO-based junction (Schottky or $p-n$ one) should have a sufficiently high rectification ratio. The hybrid structures, in which $\mathrm{ZnO}$ plays a role of the $n$-type partner of organic material or acts as a transparent conductive oxide, are also tested for photovoltaic cells [3-5].

Despite several possible applications, construction of good quality ZnO-based Schottky junctions still remains a challenging issue. This is due to the high $n$-type conductivity of $\mathrm{ZnO}$ obtained by various deposition methods [6-8]. The modeling shows that the electron concentration in $\mathrm{ZnO}$ to form a Schottky junction with a good rectification ratio should not be higher than $10^{16}-10^{17} \mathrm{~cm}^{-3}[9]$. This is crucial to achieve a low value of the diode's reverse current. Moreover, a high carrier mobility is desired to get a high forward current. Additional complication stems from a relatively high concentration of surface states in $\mathrm{ZnO}$ resulting in the Fermi level pinning. This deteriorates the rectification ratio significantly. Moreover, the Schottky barrier height (SBH)

\footnotetext{
* corresponding author; e-mail: krajew@ifpan.edu.pl
}

does not follow the difference in work functions of $\mathrm{ZnO}$ and metal. In fact, different metals have been tested as the Schottky contacts to $\mathrm{ZnO}$ [10] and the reported SBH was often independent of the metal used. It was shown that different surface pre-treatments $[11,12]$ improve the junction's current-voltage $(I-V)$ characteristics. Till now the best diodes were obtained for $\mathrm{ZnO}$ bulk crystals (mainly hydrothermally grown ones) $[13,14]$ as they have basically low carrier concentration $n \approx 10^{14}-10^{15} \mathrm{~cm}^{-3}$ due to the effective compensation with $\mathrm{Li}$ atoms.

In this work we discuss electrical properties of the $\mathrm{ZnO}$ -based Schottky junctions with silver as the Schottky metal. We apply several approaches (post-growth annealing, acceptor (nitrogen) doping via the $\mathrm{NH}_{4} \mathrm{OH}$ precursor used as an oxygen source, surface coating with a properly chosen dielectric material) to obtain the Schottky diodes with required properties.

\section{Experimental}

The $\mathrm{ZnO}$ films were grown at low temperature (between $60^{\circ} \mathrm{C}$ and $200^{\circ} \mathrm{C}$ ) by the atomic layer deposition (ALD) method using diethylzinc $\left(\mathrm{Zn}\left(\mathrm{C}_{2} \mathrm{H}_{5}\right)_{2}\right.$, DEZ) or dimethylzinc $\left(\mathrm{Zn}\left(\mathrm{CH}_{3}\right)_{2}\right.$, DMZ) and deionized water as precursors. As a nitrogen dopant the aqueous solution of $\mathrm{NH}_{3}$ (ammonia water) was applied. $\mathrm{ZnO}$ films were obtained as the result of one of two alternative double exchange reactions 


$$
\text { or } \begin{gathered}
\mathrm{Zn}\left(\mathrm{C}_{2} \mathrm{H}_{5}\right)_{2}+\mathrm{H}_{2} \mathrm{O} \rightarrow \mathrm{ZnO}+2 \mathrm{C}_{2} \mathrm{H}_{6} \\
\mathrm{Zn}\left(\mathrm{CH}_{3}\right)_{2}+\mathrm{H}_{2} \mathrm{O} \rightarrow \mathrm{ZnO}+2 \mathrm{CH}_{4} .
\end{gathered}
$$

The junctions with a silver Schottky electrode have been formed using either as grown $\mathrm{ZnO}$ layers, after their post-growth annealing, for $\mathrm{ZnO}$ doped with nitrogen, or after $\mathrm{ZnO}$ surface coating with a thin dielectric film $\left(\mathrm{HfO}_{2}\right)$, introduced between $\mathrm{ZnO}$ and the $\mathrm{Ag}$ Schottky contact. The area of the Schottky contact was about $0.7 \mathrm{~mm}^{2}$. Coating with a dielectric material was performed in a separate ALD process (at $135^{\circ} \mathrm{C}$ ) involving a reaction between tetrakis(dimethylamido)hafnium(IV) and $\mathrm{H}_{2} \mathrm{O}$.

The current-voltage characteristics (CVC) were collected using the Keithley 236 source measure unit and then processed according to the differential approach [15-17]. It bases on determination of the differential slope of the CVC curves from Eq. (3) [15]:

$$
\alpha(V)=\frac{\mathrm{d}(\lg I)}{\mathrm{d}(\lg V)}=\left(\frac{V}{I}\right)\left(\frac{\mathrm{d} I}{\mathrm{~d} V}\right),
$$

where $V$ and $I$ stand for the voltage and the current, respectively. For CVC the $\alpha$ value denotes the ratio of the static resistance $R=V / I$ to the differential resistance $\mathrm{d} R=\mathrm{d} V / \mathrm{d} I$, or the ratio of the differential conductivity $\mathrm{d} \sigma=\mathrm{d} I / \mathrm{d} V$ to the conductivity $\sigma=I / V$. The $\alpha$ value also represents the exponent in the power law dependence $I(V)=V^{\alpha}$. Similarly, the $\gamma$ parameter describing the exponential law in the form of $I(V)=\exp \left(V^{\gamma}\right)$ can be found as [15]:

$$
\gamma(V)=\frac{\mathrm{d}(\lg \alpha)}{\mathrm{d}(\lg V)}=\left(\frac{V}{\alpha}\right)\left(\frac{\mathrm{d} \alpha}{\mathrm{d} V}\right) .
$$

While processing the experimental dependences $I(V)$ using Eq. (3) one can reveal the regions of $\alpha=$ const. These are the regions where the $I(V)$ dependence is adequately approximated by a power law. Similarly, while analyzing the experimental dependences $I(V)$ using Eq. (4) one will find the regions of $\gamma=$ const. These ones correspond to the exponential behavior of $I(V)$.

In such a case the general analytical expression for the combination of power and exponential functions can be written as

$$
I(V)=\beta V^{\alpha} \exp \left(\frac{\gamma}{|\gamma|}\left(\frac{V}{V_{0}}\right)^{\gamma}\right),
$$

where $\beta$ and $V_{0}$ are constant.

According to this definition, the differential approach towards the analysis of experimental data allows one to determine the dominant carrier transport mechanism in the junction, judging from the dimensionless $\alpha$ and $\gamma$ parameters. The typical values of $\alpha$ and $\gamma$ for different known transport regimes are listed e.g. in [15].

\section{Results and discussion}

As already mentioned in Introduction, to construct a good quality $\mathrm{ZnO}$-based Schottky diode one has to over- come the problem of heavy unintentional $n$-type doping that is frequently reported for this material. Such a behavior has been attributed to a high concentration of donor type defects. Hydrogen atoms located in the interstitial positions $[18,19]$, zinc interstitials [20] and oxygen vacancies are claimed to be the most important shallow donors in $\mathrm{ZnO}$. These defects are hampering $p$-type doping in $\mathrm{ZnO}$ [21].

The free electron concentration $(n)$ in $\mathrm{ZnO}$ must be minimized to obtain a rectification in a metalsemiconductor junction. Therefore, we tested different approaches to decrease $n$ value.

For example, a post-growth annealing of a $\mathrm{ZnO}$ film (at $180^{\circ} \mathrm{C}$ and $250^{\circ} \mathrm{C}$ ) results in a drop of $n$ up to three orders of magnitude, as already reported in [22]. The rectifying effect is then observed [23]. Unfortunately, this solution is not applicable for the heterostructures containing temperature-sensitive organic materials, such as e.g. pentacene [24]. Moreover, the low thermal budget restrictions imposed to $\mathrm{ZnO}$ by the construction of a new generation of 3D memory cells built in the cross-bar architecture makes the annealing procedure inapplicable in some cases. In the latter case, the so-called back-end-of-line (BEOL) metallization, in which the metal paths are placed not only at the top, but also at the bottom of the junction, was tested by us [1]. For other purposes the "classical" front-end-of-line (FEOL) architecture may be used [25].

Another problem was observed by us when using the silver Schottky contact. When placed at the top of the structure (on the $\mathrm{ZnO}$ layer) the Schottky electrode tends to oxidize. This results in the oxygen outdiffusion from $\mathrm{ZnO}$. This leads to the formation of a very thin highly conductive layer under the $\mathrm{Ag}$ contact, which destroys the rectifying effect. As shown by us in [1], the effect can be avoided when the Schottky metallization was deposited at the bottom of the structure, primarily to $\mathrm{ZnO}$ deposition. The following contact oxidation blocked the oxygen outdiffusion from $\mathrm{ZnO}$. This allowed to obtain a diode with rectification ratio of $10^{8}$ (at $\pm 2 \mathrm{~V}$ ) and current density of about $10^{4} \mathrm{~A} \mathrm{~cm}^{-2}$. Such parameters were suitable for a selector application in the cross-bar memories [1]. The obtained diode revealed the ideality factor $\eta \approx 2.06$ and is still competitive with the ones constructed for analogous purposes by industrial companies [26].

In the present work we test the "traditional" architecture (with the top Schottky contact) for the sensing applications of $\mathrm{ZnO}$-based structures [27, 28]. We first tested the applicability of $\mathrm{ZnO}$ films doped (compensated) with nitrogen applying the $\mathrm{NH}_{4} \mathrm{OH}$ precursor. Nitrogen acts as a $p$-type dopant in $\mathrm{ZnO}$ (with high ionization energy of about $1.3 \mathrm{eV}$ [29]), effectively compensating the free electron concentration [28]. Figure 1 shows that nitrogen is built-in uniformly into the host material. We observed that $\mathrm{N}$-doping results in a substantial drop of $n$ down to the values of about $10^{14} \mathrm{~cm}^{-3}$. We also noticed a decrease of diode reverse current with increasing nitrogen 
concentration (see Fig. 2). However, if $\mathrm{N}$ concentration exceeds $2-3 \%$ we observe a decrease of electrons' Hall mobility due to the enhanced scattering processes at ionized impurities. The forward (driving) current is then decreasing (see Fig. 2).

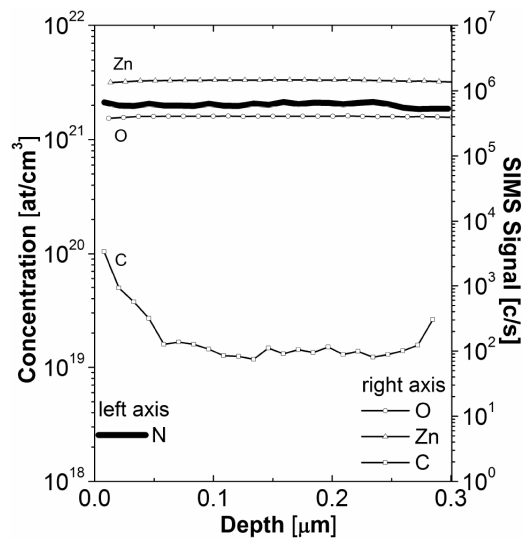

Fig. 1. Secondary ion mass spectroscopy (SIMS) profile of $\mathrm{ZnO}: \mathrm{N}$ thin film with doping level of about $2.3 \%$. As can be seen, nitrogen is uniformly distributed in the $\mathrm{ZnO}$.

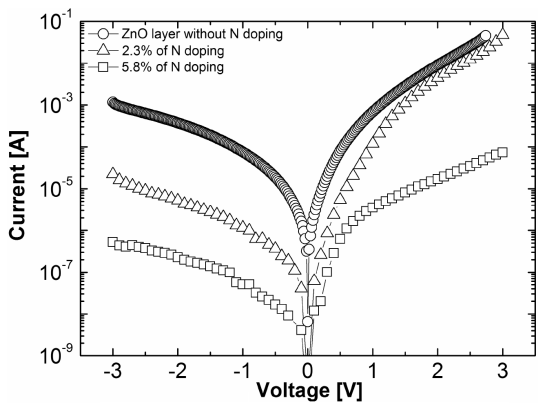

Fig. 2. Current-voltage characteristics of $\mathrm{ZnO} / \mathrm{Ag}$ junctions for undoped $\mathrm{ZnO}$ (circles), $\mathrm{ZnO}: \mathrm{N}$ with $2.3 \%$ of nitrogen (triangles) and $\mathrm{ZnO}: \mathrm{N}$ with $5.8 \%$ of nitrogen (squares).

An "optimally doped" $\mathrm{ZnO}: \mathrm{N} / \mathrm{Ag}$ diode reveals the rectification ratio $2.1 \times 10^{3}$ at $\pm 3 \mathrm{~V}$ and ideality factor $\eta \approx 2.65$ according to the well known thermionic emission theory, defining the thermionic emission current $I_{\mathrm{th}}$ as:

$$
I_{\mathrm{th}}=I_{\mathrm{s}}\left[\exp \left(\frac{q\left(V-I_{\mathrm{th}} R_{\mathrm{s}}\right)}{\eta k T}\right)-1\right],
$$

where $R_{\mathrm{s}}$ and $I_{\mathrm{s}}$ denote a diode series resistance and a saturation current, respectively. $I_{\mathrm{s}}$, from which a Schottky barrier height $\varphi_{\mathrm{B}}$ can be estimated, is given by

$$
I_{\mathrm{s}}=A A^{*} T^{2} \exp \left(\frac{-q \varphi_{\mathrm{B}}}{k T}\right),
$$

where $A$ is the contact area (approximately $0.7 \mathrm{~mm}^{2}$ ) and $A^{*}$ denotes the effective Richardson constant (theoretically for $\mathrm{ZnO} A^{*}=32 \mathrm{~A} \mathrm{~K}^{-2} \mathrm{~cm}^{-2}$ ). As derived from these formulae, $\varphi_{\mathrm{B}}$ for the $\mathrm{ZnO}$ :N-based diode is about $0.7 \mathrm{eV}$. A similar value of $\varphi_{\mathrm{B}}$ has been reported for the Schottky junctions on bulk $\mathrm{ZnO}[13,28]$.

From the low temperature Hall effect measurements we found that scattering on ionized impurities and grain boundaries are the two dominant (and competitive) scattering mechanisms in $\mathrm{ZnO}$ films. The first one is especially efficient in the case of epitaxial films with an electron concentration $n \approx 10^{18} \mathrm{~cm}^{-3}$ and can be described by the Brooks-Herring theory, according to which the Hall mobility $\mu$ and temperature $T$ stay in the relation of $\mu \propto T^{3 / 2}$ [30]. The second one (applicable probably to the nitrogen-compensated films as well) seems to dominate in polycrystalline films with the grain diameter of about $20-25 \mathrm{~nm}$ and low $\left(n \approx 10^{16} \mathrm{~cm}^{-3}\right)$ carrier concentration. Further details will be given elsewhere.

Further increase in the $\mathrm{ZnO} / \mathrm{Ag}$ junction's rectification ratio (up to $10^{4}-10^{5}$ at $\pm 2 \mathrm{~V}$ ) - see Fig. 3 - is possible for the $\mathrm{ZnO}$ film coated with a thin layer of high- $k$ dielectric material, e.g. $\mathrm{HfO}_{2}$. The investigations presented in [31] proved that the optimal thickness of covering is about $2.5 \mathrm{~nm}$. The main advantage of such an approach is band bending regulation.

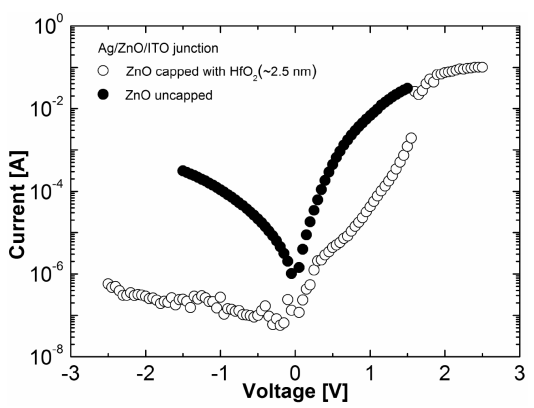

Fig. 3. Influence of $\mathrm{HfO}_{2}$ capping layer on the $I-V$ characteristics of $\mathrm{Ag} / \mathrm{ZnO} / \mathrm{ITO}$ structure with the top Ag Schottky contact: diode with uncoated $\mathrm{ZnO}$ (black circles) and coated with $\approx 2.5 \mathrm{~nm}$ of $\mathrm{HfO}_{2}$ (white circles).

Thin film of $\mathrm{HfO}_{2}$ deposited on the $\mathrm{ZnO}$ layer still allows carriers' flow through the junction and does not affect importantly $n$ concentration of the underlying $\mathrm{ZnO}$ film. For that reason aluminum oxide $\left(\mathrm{Al}_{2} \mathrm{O}_{3}\right)$ should not be used for capping as $\mathrm{Al}$ effectively provides free electrons to the host $\mathrm{ZnO}$ film [32]. The $\mathrm{HfO}_{2}$-coated diodes reveal the $\mathrm{SBH}$ reaching $0.5-0.7 \mathrm{eV}$.

After constructing of Schottky diodes we tested their sensing properties. We have done this for the junctions built in the planar architecture, where the current is parallel to the film surface. This allows to functionalize the structure easily. As presented in Fig. $4 \mathrm{a}-\mathrm{c}$, the reverse current of the diode evidently increases under the exposition to a droplet of acetone $\left(\mathrm{C}_{3} \mathrm{H}_{6} \mathrm{O}\right)$. In fact, the effect disappears after $\mathrm{C}_{3} \mathrm{H}_{6} \mathrm{O}$ evaporation, which takes about $5 \mathrm{~min}$. Interestingly, the diode-based sensor resets at room temperature (Fig. 4b) without any special treat- 


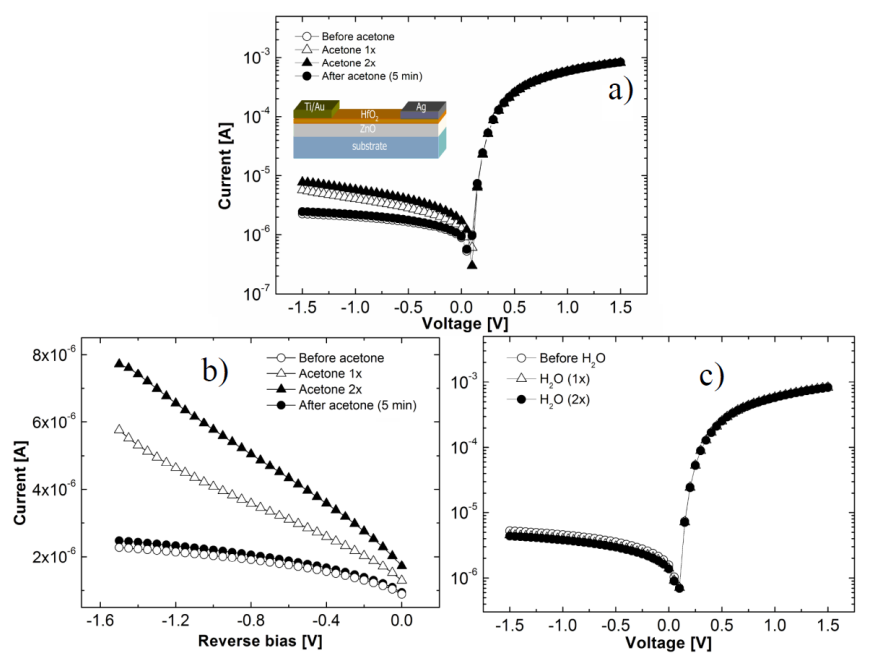

Fig. 4. Sensing properties of a planar $\mathrm{ZnO}$-based Schottky diode: after exposition to acetone $\left(\mathrm{C}_{3} \mathrm{H}_{6} \mathrm{O}\right)$ in semilogarithmic scale (a), linear scale (b) and after exposition to $\mathrm{H}_{2} \mathrm{O}$ in semilogarithmic scale (c). The junction's scheme is presented in the inset to Fig. 4a.

ment and shows almost no response when treated with water (see Fig. 4c).

The junctions' modeling performed according to the differential approach, presented in Fig. $5 \mathrm{a}-\mathrm{c}$ showed that the differential slope $\alpha$ demonstrates various behaviors under introducing the $\mathrm{HfO}_{2}$ interlayer (Fig. 5a). In all cases except the one when the thickness of the $\mathrm{HfO}_{2}$ interlayer $(d)$ is $2.5 \mathrm{~nm}$, there is the restriction of the current imposed by the contact emission at high bias $(>1 \mathrm{~V})$.

The evidence of this is the value of $\alpha$ : in these cases $\alpha \leq 1$ [16]. Only the case with $d=2.5 \mathrm{~nm}$ shows the values of $\alpha=1.5$ at high bias, corresponding to the bimolecular recombination when concentration of major $(n)$ and minor $(p)$ charge carriers is almost equal $[16,33,34]$. So, there is the thickness of $\mathrm{HfO}_{2}$ layer $d=2.5 \mathrm{~nm}$, which favors a good injection of both types of charge carriers into the $\mathrm{ZnO}$ film.

The differential images of $\alpha(V)$ and $\gamma(V)$ for low bias $(V<1 \mathrm{~V})$ are shown in Fig. 5b and c. The initial range of $\alpha(V)(0<V<0.1 \mathrm{~V})$ shows an ideal diode behaviour where $\alpha=k T / \eta e V$ and $\gamma=1$ [35]. The next ranges of curve have two sections which can be approximated by the reciprocal exponent $(0.2 \mathrm{~V}<V<0.4 \mathrm{~V}$ with $\gamma=1)$ and the reciprocal square root exponent $(0.4 \mathrm{~V}<V<$ $0.8 \mathrm{~V}$ with $\gamma=-0.5)$.

\section{Summary and conclusions}

The properties of $\mathrm{ZnO} / \mathrm{Ag}$ Schottky junctions have been discussed. Zinc oxide films were grown by the atomic layer deposition method using either diethylzinc or dimethylzinc and deionized water as precursors. The films were deposited at low temperature between $60^{\circ} \mathrm{C}$ and $200^{\circ} \mathrm{C}$. We have shown that: post-growth annealing,
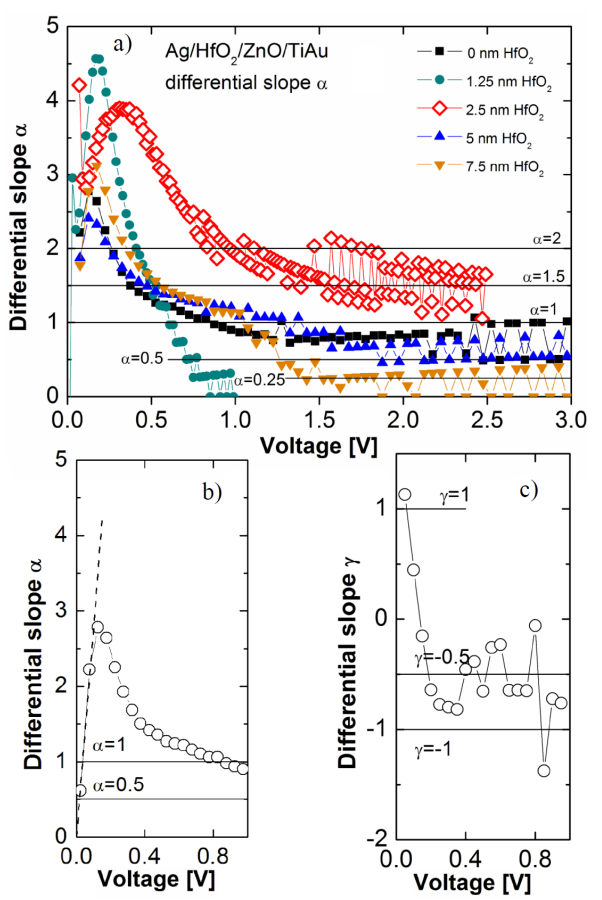

Fig. 5. The differential slope $(\alpha)$ versus voltage for the $\mathrm{ZnO} / \mathrm{Ag}$ Schottky diodes with different thickness of $\mathrm{HfO}_{2}$ interlayer (a). The $\alpha(V)(\mathrm{b})$ and $\gamma(V)$ (c) dependences show an ideal diode behavior for the low voltage range $(0<V<0.1 \mathrm{~V})$ according to Eq. (3) and Eq. (4).

ZnO doping with nitrogen and the layers' surface covering with a thin dielectric film $\left(\mathrm{HfO}_{2}\right)$ before introducing the silver electrode result in the higher rectification ratio of the Schottky junction.

The best results (the highest rectification ratio) have been achieved in the diode constructed in the "inversed" architecture (with the bottom Schottky electrode). For the FEOL architecture the best results have been obtained for a $\mathrm{ZnO}$ surface coated with a thin dielectric layer.

Modeling of the structures, based on the thermionic emission theory and differential approach gives the ideality factors $\eta \approx 2$. The sensing application of the obtained junctions has been demonstrated.

\section{Acknowledgments}

The research was partially supported by the European Union within European Regional Development Fund, through grant Innovative Economy (POIG.01.01.02-00$-008 / 08)$ and by grant of the National Science Center of Poland (1669/B/H03/2011/40).

\section{References}

[1] N. Huby, G. Tallarida, M. Kutrzeba, S. Ferrari, E. Guziewicz, Ł. Wachnicki, M. Godlewski, Microelectron. Eng. 85, 2442 (2008). 
[2] E. Katsia, N. Huby, G. Tallarida, B. Kutrzeba-Kotowska, M. Perego, S. Ferrari, F.C. Krebs, E. Guziewicz, M. Godlewski, V. Osinniy, G. Luka, Appl. Phys. Lett. 94, 143501 (2009).

[3] P.I. Stakhira, G.L. Pakhomov, V.V. Cherpak, D. Volynyuk, G. Luka, M. Godlewski, E. Guziewicz, Z.Yu. Hotra, Centr. Eur. J. Phys. 8, 798 (2010).

[4] M. Godlewski, E. Guziewicz, G. Łuka, T. Krajewski, M. Łukasiewicz, Ł. Wachnicki, A. Wachnicka, K. Kopalko, A. Sarem, B. Dalati, Thin Solid Films 518, 1145 (2009).

[5] A. Bosio, N. Romeo, S. Mazzamuto, V. Cannevari, Prog. Cryst. Growth Character. Mater. 52, 247 (2006).

[6] K.T. Roro, G.H. Kassier, J.K. Dangbegnon, S. Sivaraya, J.E. Westraadt, J.H. Neethling, A.W.R. Leitch, J.R. Botha, Semicond. Sci. Technol. 23, 055021 (2008).

[7] J. Lim, K. Shin, C. Lee, J. Mater. Sci. 39, 3195 (2004).

[8] G. Gordillo, C. Calderón, Solar Energy Mater. Solar Cells 69, 251 (2001).

[9] M. Pra, G. Csaba, C. Erlen, P. Lugli, J. Comput. Electron. 7, 146 (2008).

[10] K. Ip, G.T. Thaler, H. Yang, S.Y. Han, Y. Li, D.P. Norton, S.J. Pearton, S. Jang, F. Ren, J. Cryst. Growth 287, 149 (2006).

[11] Q.L. Gu, C.C. Ling, X.D. Chen, C.K. Cheng, A.M.C. Ng, C.D. Beling, S. Fung, A.B. Djurišić L.W. Lu, G. Brauer, H.C. Ong, Appl. Phys. Lett. 90, 122101 (2007).

[12] O. Hamad, G. Braunstein, H. Patil, N. Dhere, Thin Solid Films 489, 303 (2005).

[13] H. Kim, H. Kim, D.-W. Kim, J. Appl. Phys. 108 074514 (2010).

[14] Vl. Kolkovsky, L. Scheffler, E. Hieckmann, E.V. Lavrov, J. Weber, Appl. Phys. Lett. 98, 082104 (2011).

[15] P. Smertenko, L. Fenenko, L. Brehmer, S. Schrader, Adv. Coll. Interface Sci. 116, 255 (2005).

[16] R. Ciach, Yu. Dotsenko, V. Naumov, A. Shmyryeva, P. Smertenko, Solar Energy Mater. Solar Cells $\mathbf{7 6}$, 613 (2003).

[17] V. Mikhelashvili, G. Eisenstein, V. Garber, S. Fainleib, G. Bahir, D. Ritter, V. Orenstein, A. Peer, J. Appl. Phys. 85, 6873 (1999).

[18] C.G. Van de Walle, Phys. Rev. Lett. 85, 1012 (2000).
[19] S.J. Pearton, D.P. Norton, K. Ip, Y.W. Heo, T. Steiner, Superlatt. Microstruct. 34, 3 (2003).

[20] M. Gomi, N. Oohira, K. Ozaki, M. Koyano, Jpn. J. Appl. Phys. 42, 481 (2003).

[21] A. Janotti, C.G. Van de Walle, Rep. Prog. Phys. 72, 126501 (2009).

[22] T. Krajewski, E. Guziewicz, M. Godlewski, L. Wachnicki, I.A. Kowalik, A. Wojcik-Glodowska, M. Łukasiewicz, K. Kopalko, V. Osinniy, M. Guziewicz, Microelectron. J. 40, 293 (2009).

[23] T.A. Krajewski, G. Luka, L. Wachnicki, M.I. Lukasiewicz, A.J. Zakrzewski, B.S. Witkowski, R. Jakiela, E. Lusakowska, K. Kopalko, B.J. Kowalski, M. Godlewski, E. Guziewicz, Phys. Chem. Solid State 12, 224 (2011).

[24] G. Łuka, T. Krajewski, Ł. Wachnicki, A. Szczepanik, J.D. Fidelus, A. Szczerbakow, E. Łusakowska, K. Kopalko, E. Guziewicz, M. Godlewski, Acta Phys. Pol. A 114, 1229 (2008).

[25] K. Ishimaru, Solid-State Electronics 52, 1266 (2008).

[26] Y. Sasago, M. Kinoshita, T. Morikawa, K. Kurotsuchi, S. Hanzawa, T. Mine, A. Shima, Y. Fujisaki, H. Kume, H. Moriya, N. Takaura, K. Torii, in: Symposium on VLSI Technology, Honolulu 1998.

[27] T. Anderson, F. Ren, S. Pearton, B.S. Kang, H.-T. Wang, C.-Y. Chang, J. Lin, Sensors 9, 4669 (2009).

[28] S. Liang, H. Sheng, Y. Liu, Z. Huo, Y. Lu, H. Shen, J. Cryst. Growth 225, 110 (2001).

[29] J.L. Lyons, A. Janotti, C.G. Van de Walle, Appl. Phys. Lett. 95, 252105 (2009).

[30] K. Ellmer, J. Phys. D, Appl. Phys. 34, 3097 (2001).

[31] T.A. Krajewski, G. Luka, S. Gieraltowska, A.J. Zakrzewski, P.S. Smertenko, P. Kruszewski, L. Wachnicki, B.S. Witkowski, E. Lusakowska, R. Jakiela, M. Godlewski, E. Guziewicz, Appl. Phys. Lett. 98 263502 (2011).

[32] G. Luka, L. Wachnicki, B.S. Witkowski, T.A. Krajewski, R. Jakiela, E. Guziewicz, M. Godlewski, Mater. Sci. Eng. B 176, 237 (2011).

[33] M. Lampert, P. Mark, Current Injection in Solids, Academic Press, New York 1970.

[34] R. Baron, J.W. Mayer, in: Semiconductors and Semimetals, Academic Press, New York 1970, p. 201.

[35] S.M. Sze, Physics of Semiconductor Devices, 3 ed., Wiley-Interscience, New York 2006. 